\title{
Submitted for
}

Proceedings of the XI International Conference on Small-Angle Scattering Upton, NY

May 17-20, 1999

\section{PHASE BEHAVIOR OF BLENDS OF LINEAR AND BRANCHED POLYETHYLENES ON MICRON-LENGTH SCALES VIA ULTRA-SMALL-ANGLE NEUTRON SCATTERING (USANS)}

\section{M. Agamalian ${ }^{1,2}$, R. G. Alamo ${ }^{3}$, J. D. Londono $0^{1,4}$ L. Mandelkern ${ }^{5}$, and G. D. Wignall'}

'Solid State Division, Oak Ridge National Laboratory, Oak Ridge, TN 37831-6393

'Present Address: Argonne National Laboratory, 9700 S. Cass Ave., Argonne, IL 60439

'Florida Agricultural and Mechanical University and Florida State University College of Engineering, Tallahasse, FL 32310

${ }^{4}$ Present Address: DuPont Central R\&D Experimental Station, Wilmington, DE 19880-0323

${ }^{5}$ Institute of Molecular Bio-Physics, Florida State University, Tallahasse, FL 32306

\author{
"The submitted manuscript has been authored \\ by a contractor of the U.S. Government under \\ contract No. DE-AC05-960R22464. \\ Accordingly, the U.S. Government retains a \\ nonexclusive, royalty-free license to publish \\ or reproduce the published form of this \\ contribution, or allow others to do so. for U.S. \\ Government purposes." \\ prepared by \\ SOLID STATE DIVISION \\ OAK RIDGE NATIONAL LABORATORY \\ Managed by \\ LOCKHEED MARTIN ENERGY RESEARCH CORP. \\ under \\ Contract No. DE-AC05-960R22464 \\ with the \\ U.S. DEPARTMENT OF ENERGY \\ Oak Ridge, Tennessee
}

May 1999 
PHASE BEHAVIOR OF BLENDS OF LINEAR AND BRANCHED POLYETHYLENES ON MICRON LENGTH-SCALES VIA ULTRA-SMALL ANGLE NEUTRON SCATTERING (USANS)

M. M. Agamalian (a), R. G. Alamo (b), J. D. Londono (a,c), L. Mandelkem (d) and G. D. Wignall (a).

(a): Solid State Division, Oak Ridge National Laboratory*, Oak Ridge, TN 37831-6393

(b): Florida Agricultural and Mechanical University and Florida State University College of Engineering, Department of Chemical Engineering, Tallahassee, FL 32310

(c): Current Address: DuPont Central R \& D Experimental Station, Wilmington DE 19880-0323

(d): Institute of Molecular Bio-physics, Florida State University, Tallahassee, FL 32306

Abstract: SANS experiments on blends of linear, high density (HD) and long chain branched, low density (LD) polyethylenes indicate that these systems form a one-phase mixture in the melt. However, the maximum spatial resolution of pinhole cameras is $-10^{3} \AA$ and it has therefore been suggested that data might also be interpreted as arising from a bi-phasic melt with large a particle size $(-1 \mu \mathrm{m})$, because most of the scattering from the different phases would not be resolved. We have addressed this hypothesis by means of USANS experiments, which confirm that HDPE/LDPE blends are homogenous in the melt on length scales up to $20 \mathrm{pm}$. We have also studied blends of HDPE and short-chain branched linear low density polyethylenes (LLDPEs), which phase separate when the branch content is sufficiently high. LLDPEs prepared with Ziegler-Natta catalysts exhibit a wide distribution of compositions, and may therefore be thought of as a "blend" of different species. When the composition distribution is broad enough, a fraction of highly branched chains may phase separate on pm-length scales, and USANS has also been used to quantify this phenomenon.

*Managed by Lockheed Martin Energy Research Corp. under contract DE-AC05-84OR2140 for the U. S. Department of Energy. 
Introduction: Polyethylene (PE) is produced in many forms, each of which has different properties resulting from variations in structure. High density polyethylene (HDPE) is the most crystalline form, because the chains contain very little branching. Typical low density polyethylenes (LDPE) contain both short chain branches (I-3 per 100 backbone carbon atoms), as well as long chain branches (0.1-0.3 per 100 backbone carbon atoms). Linear low density polyethylene (LLDPE) contains only short-chain branches, but can have a wide range of branch contents, depending on the catalyst and concentration of added (alpha olefin) comonomer. The properties of the individual species can be altered by mixing the components and blends of HDPE, LDPE and LLDPE are widely used commercially. However, understanding of the mechanical and melt flow properties of such blends is handicapped by the absence of a consensus concerning the melt miscibility of the components. For example, different views have been expressed in the literature ranging from complete homogeneity in the melt (Alamo et al., 1994, 1997) to liquid-liquid phase segregation (Schipp, 1996, Barham, 1968, Hill, 1991 and co-workers) for HDPE/LDPE mixtures. SANS can supply information on melt homogeneity of polymer blends via the contrast achieved by deuteration, and this technique has been used extensively to study the melt compatibility, solid state morphology (Wignall et al., 1996) and thermodynamics (Londono, 1995, Krishnamoorti, 1994, Graessley, 1994, Nesarikar, 1994 and co-workers) of mixtures of linear and branched polyolefins, including HD, LD and LLDPEs.

Pinhole SANS data indicate that for HDPELDPE blends with molecular weights $\sim 10^{5}$, the melt are homogenous (Alamo et al., 1994), after accounting for $\mathbf{H}^{1} / \mathbf{D}^{2}$ isotope effects (Londono et al., 1994). Similarly, mixtures of HDPE and LLDPE are homogenous in the melt when the branch content is low (i.e. $<4$ br./100 backbone carbons). However, when the branch content is higher (> $10 \mathrm{br} . / 100 \mathrm{C}$ ), the blends phase separate (Alamo et al., 1997). However, Schipp and co-workers (1996) have asserted that these experiments do not provide unambiguous evidence for a I-phase (homogenous) melt for HDPE/LDPE blends, as the data might also be interpreted as arising from a bi-phasic melt with a very large particle size. 
SANS experiments (Alamo et al., 1994) were performed with a minimum value of the momentum transfer, $Q_{\min }=4 \pi \lambda^{-1} \sin \theta-0.004 \AA^{-1}$ (where $A$ is the wavelength and 29 is the angle of scatter), so the maximum spatial resolution is therefore $D \sim 2 \pi / Q_{\min } \sim 1500 \AA$. Thus, if the domains had pm-size dimensions, much of the scattering from the different phases would be exhibited at $Q$-values $<10^{-3} \AA^{-1}$. We have addressed this hypothesis via a new ultra-high resolution (USANS) instrument (Agamalian et al., 1997), lowers $Q_{\min }$ to $\sim 3 \times 10^{-5} \AA^{-1}$ and increases the spatial resolution to $\sim 20 \mu \mathrm{m}$. These experiments confirm that blends of HDPE and LDPE are homogenous and demonstrate that the phase separated blends have extremely high cross sections $\left(\mathrm{d} \Sigma / \mathrm{d} \Omega \sim 10^{8} \mathrm{~cm}^{-1}\right.$ at $\left.\mathrm{Q}-10^{-5} \mathrm{~A}^{-1}\right)$, which is six orders of magnitude higher than for homogenous systems.

Experimental: The HDPE and LDPEs are identical to those used in previous works (Alamo et al., 1994, 1997), using the same nomenclature for the polymer components as in previous publications. A 75/25 blend of low density polyethylene (LDPE-3H) and deuterated DHPE (HDPE4D) was prepared and would be expected to be homogeneous in the melt, based on previous SANS experiments. As example of a phase separated blend, a 75/25 mixture of a highly branched hydrogenated polybutadiene (HPB-H35 with 10.6 mol\% of ethyl branches ) and linear polyethylene was also prepared. The blends were made as described previously and the percentage of each component in this blend were as follows, $75 \%$ of hydrogenated polybutadiene HPB-H35, 15\% of linear protonated (HDPE-1) and 10\% of linear deuterated polyethylene (HDPE4D). By contrast, the amount of deuterated material (25\%) was much higher $(2.5 x)$ in the HDPE/LDPE blend to enhance the scattering from any morphological features present. For studies of heterogeneous LLDPEs, a commercial ethylene-hexene-1 (EH) copolymer was dissolved in p-xylene and allowed to cool to room temperature, where the high molecular weight, lightly branched material crystallizes out of the solution. The lower molecular weight, highly branched component (- 8\%) was recovered from the filtrate and the original EH-copolymer, xylene extract and filtrate were each blended with $20 \%$ deuterated HDPE. 
Pinhole SANS data were collected on the W. C. Koehler 30m SANS facility (Koehler, 1986) at the Oak Ridge National Laboratory via a $64 \times 64 \mathrm{~cm}^{2}$ area detector with cell size $\sim 1 \mathrm{~cm}^{2}$ and sample-detector distances of $10 \mathrm{~m}$ and $19 \mathrm{~m}$. The beamstop size (diameter) was $4 \mathrm{~cm}$, and the wavelength $(A)$ was $4.75 \AA$. The data were corrected on a cell-by-cell basis for the efficiency variation of the detector, instrumental (beam blocked) backgrounds and also the intensities of the corresponding sample cells with quartz windows, which formed only a minor perturbation. The net intensities were converted to an absolute $( \pm 3 \%)$ differential cross section per unit sample volume $\left[\mathrm{d} \Sigma / \mathrm{d} \Omega(Q)\right.$ in units of $\left.\mathrm{cm}^{-}\right]$by comparison with pre-calibrated secondary standards (Wignall and Bates, 1986), and radially averaged to give a $Q$-range of $1.5 \times 10^{-3}<Q<0.06 \AA^{-1}$. The USANS measurements were carried out on a Bonse-Hart Double-Crystal Diffractometer equipped by triple-bounce Si(111) channel-cut crystals, modified by cutting an additional groove for a cadmium absorber (Agamalian, Wignall and Triolo, 1997), which reduces the wings of the rocking curve by three orders of magnitude. The Q-range $\left(3 \times 10^{-5}<Q<2 \times 10^{-3} \AA^{-1}\right)$ was chosen to overlap with the pinhole SANS measurements and the data normalized via the neutron beam monitor to correct for drifts in the incident beam intensity. After subtracting the instrumental backgrounds, the net intensities were divided by the sample thickness and transmission coefficient, which was measured by the monitor detector located behind the analyzer crystal [fig. (1)]. Further details of the data treatment and absolute calibration procedures are given by Agamalian et al. (1999).

Results and Discussion (1) Blends of Linear and Branched Polyethylenes: When a sample is placed between the analyzer and monochromator in a USANS experiment [fig. (1)], small-angle scattering "spreads" the beam, and this is reflected in the excess intensity observed for $\mathbf{Q}>\mathbf{Q}_{\min }$ " $3 \times 10^{-5} \AA^{-1}$, as shown in fig. (2) for the three samples at $160^{\circ} \mathrm{C}$. It may be seen that the signal from the phase separated (HDPE/HPB) blend is orders of magnitude higher than the scattering from the HDPE-D/LDPE sample and unlabeled LDPE "blank". Moreover, the latter data are virtually superimposed on the signal produced by the empty cell, with no sample at all. Thus, the cross section of these two samples close to the detection limit of current USANS instrumentation 
and the small net signal which is barely resolved beyond the experimental scatter for the HDPE/LDPE sample is virtually the same for the LDPE blank. Thus, it must arise from urn-sized heterogeneities (catalyst residues, dirt, antioxidants etc.), which are extrinsic to the blend morphology (Renninger et al., 1995). The scattering from the phase separated HPB/HDPE is clearly resolved by USANS, despite the fact that the level of deuterium labeling is lower (2.5x) than in the HDPULDPE blend. Had this sample been phase separated on pm-sized length scales, as affirmed by Schipp and co-workers (1996), the cross section would have been well above the USANS detection limit and have greatly exceeded the signal of the LDPE homopolymer blank. Thus, the USANS results are consistent with the conclusions that the HDPE-D/HPB sample is phase separated in, the melt and the HDPE-D/LDPE is homogenous, as previously indicated by pinhole SANS measurements.

This conclusion is further reinforced combining the USANS and pinhole SANS data from HDPE/HPB blend, though before this can be accomplished, corrections must be applied for instrumental resolution effects, which "smear" the data by integrating over a finite range of angles. In general, such effects are much smaller for SANS experiments than for USANS, because the former are taken with pinhole collimation, whereas USANS facilities use long-slit geometry, where smearing effects are much larger. The main component of the distortion arises from the large range of angles $(\Delta \theta)$ in the "vertical" plane and collimation "desmearing" was accomplished by the Sabine-Bertram model, combined with the Lake technique, which allows USANS data, collected in slit geometry to be transferred to point geometry (Agamalian et al., 1999). The data are "smeared" by integrating over a finite range of angles, $\Delta \theta$, and as $\Delta \theta$ is relatively independent of $\theta$, the angular uncertainty $(\Delta \theta / \theta)$ is greater as $\theta-0$, so the effects of smearing is more pronounced at smaller angles. This can alter the ratio of the data at low $\left(10^{-5} \AA^{-1}\right)$ and high $\left(10^{-3} \AA^{-1}\right) \mathrm{Q}$-values by up to two orders of magnitude [fig. (3)]. The fact that the desmeared data overlap smoothly with the independently calibrated pinhole SANS data, with no adjustable scale factors, indicates the consistency of the data treatment procedures. 
The intensity of macromolecular scattering measured from the HDPULDPE sample by the $30 \mathrm{~m}$ SANS facility is lower than the USANS detection limit $\left(-10^{3} \mathrm{~cm}-{ }^{-}\right)$, and thus the SANS scattering curve was extrapolated to the USANS region by fitting to the de Gennes' random phase approximation, which has been widely used to describe partially deuterated homogenous blends (Alamo et al., 1994). It may be seen that there is a dramatic difference (approximately six orders of magnitude) between the zero-Q cross sections of the HDPE/HPB and HDPE/LDPE samples.

It is clear from fig (3) that USANS can clearly demonstrate phase separation on pm-sized length scales when present (e.g. for HDPE/HPB). The fact that no such signal is detected from the HDPULDPE sample, despite the fact that the level of deuteration, and hence the neutron scattering contrast, is higher (25\%) than for HDPE/HPB (10\%), clearly shows that an appreciable concentration of pm-sized phase separated domains is not present in this blend.

Results and Discussion (2): Heterogeneous LLDPEs: For LLDPEs prepared with heterogeneous-type Ziegler-Natta catalysts, the multi-site nature of catalysts leads to a wide distribution of chain compositions, with the low molecular weight chains exhibiting the most branching. Heterogeneous LLDPE may therefore be thought of as a "blend" of different species and when the composition distribution is broad enough the multicomponent system can, in principle, phase separate. Thus, even when the average branch content is low $(-2 \mathrm{br} . / 100 \mathrm{C})$, a fraction of highly branched chains (> $10 \mathrm{br} . / 100 \mathrm{C}$ ), which are incompatible with the lightly branched molecules may phase separate, as first suggested by Mirabella and Ford (1987), based on scanning electron microscopy (SEM) investigations of the solid state. Subsequently, Nesarikar and Crist (1994) performed a thermodynamic calculation of the equilibrium melt state as a function Of the distribution of chain branching, which predicted a second phase consisting of highly branched amorphous material. The volume fraction $\left(f \sim 10^{-2}\right)$ was in reasonable agreement with the SEM findings.

SANS has previously been used to examined the structure.of such materials in the liquid state (Wignall et al., 1996), and a fraction of deuterated linear polymer (20\%) was added in order to manifest the melt structure. Based on previous studies, the linear material should be 
incompatible with the minority phase, but should mix homogeneously with the predominantly low branched matrix. Thus, the addition of HDPE-D should provide SANS contrast between the phases, without perturbing the predicted two-phase morphology.

Fig. (4) shows a log-log plot of the cross section in the melt $\left(T=160^{\circ} \mathrm{C}\right)$ for the original ethylene-hexene LLDPE, along with the same sample after xylene extraction (after blending with 20 wt.\% linear D-HDPE to provide SANS contrast). The existence of multiple phases is reflected in the two regions where the scattering curve varies as $Q^{-3.8}$ and $Q^{-1.7}$ for the lowest and highest Q-values respectively. These values are close to the Porod limit $\left(Q^{-4}\right)$, expected for separate phases with sharp boundaries, and to the Gaussian coil limit $\left(Q^{-2}\right)$, expected for random coil molecules. It may be seen that the extraction procedure, which removes the highly branched and low molecular weight components, only changes the scattering at the lowest Q-values, and that the data superimpose over most of the range $\left(Q>0.01 A^{-1}\right)$. Thus, xylene extraction effectively removes the component, which varies as $Q^{-4}$. The original LLDPE has an average branch content of 1.4 mole \% butyl branches (or $1.4 \mathrm{br} . / 100 \mathrm{C}$ ), though a small fraction of the distribution will be highly branched, and chains with $>10 \mathrm{br} . / 100 \mathrm{C}$ should phase separate from the lightly branched matrix. As the disperse phase (volume fraction, $f \sim 10^{-2}$ ) is incompatible with lightly branched chains, it is to be expected that it is also incompatible with the linear (deuterated) material which is added to provide SANS contrast. If these domains consisted of particles with relatively sharp boundaries, this would naturally give rise to the $Q^{-4}$ variation observed in the low-Q (Porod) limit. Conversely, it is well known that lightly branched material is compatible with linear HDPE-D, so the matrix should consist of an homogenous mixture of HDPE-D and LLDPE-H chains, and such a morphology would also give rise to the observed $Q^{-2}$ variation over most of the $Q$-range. Thus the 2-phase hypothesis accounts qualitatively for the general features of the scattering.

The volume fraction of the disperse phase can be estimated from the SANS invariant:

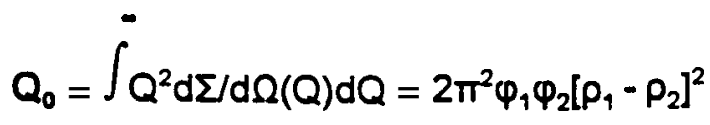


where $\varphi_{1}, \varphi_{2}, \rho_{1}$ and $\rho_{2}$ are the volume fractions and neutron scattering length densities of the two phases respectively. The scattering from the minority (disperse) phase is manifested below $Q \sim$ $0.01 \mathbf{A}^{\prime}$, where it is superimposed on the cross section of the majority of the sample consisting of a homogenous mixture of HDPE-D and LLDPE-H. This coherent "background" was removed by subtracting the cross section of the xylene-extracted material, which superimposes on the Scattering of the original blend for $Q>0.01 \AA^{-1}$. In order to estimate the portion of the area' below the minimum $\mathrm{Q}$-value $\left(0.004 \mathrm{~A}^{-1}\right)$, it was assumed that the missing low-Q data follow the Guinier approximation, to give an estimate of $f=0.021$ for the original sample via the equation (1).

The xylene-extract formed $8 \%$ of the original polymer, $2 \%$ of which was phase separated, and thus we might expect that the volume fraction of the disperse phase in the xylene extract would be $f=0.20$, when blended with $20 \%$ HDPE-D. Invariant analysis'leads to $f=0.20$, after correction for the coherent "background" from scattering from the homogeneously mixed material (Wignall et al., 1996). The close agreement between these estimates is probably fortuitous in view of the fact that the scattering from particles with dimensions $>1500 \AA$ appears at $Q$-values below the resolution limit of the experiment. However, USANS allows us to fill in the missing portion of the data and improve the estimate of $f$. Figure (5) shows the overlap of the pinhole SANS and USANS data and invariant analysis of the combined curve leads to a volume fraction of the disperse phase of $f=0.30$. This may be compared with the previous determination $(f-0.20$ ), derived from the pinhole SANS data. Thus USANS substantially improves the estimate of $f$ by filling in the previously inaccessible portion of the SANS invariant.

Acknowledgments: The research at Oak Ridge was supported by the Division of Materials Sciences, U. S. Department of Energy under Contract No. DE-AC05-960R22464 with Lockheed Martin Energy Research Corporation and in part by appointment to the Oak Ridge National Laboratory postdoctoral research associates program administered jointly by the Oak Ridge National Laboratory and the Oak Ridge Institute for Science and Education. The work at Florida State was supported by the National Science Foundation Polymer Program (DMR 94-19508). 


\section{References:}

Agamalian, M. M., Wignall, G. D. \& R. Triolo, (1997), J. Appl. Cryst., 30, 345-352.

Agamalian, M. M., Alamo, R. G.. Londono, J. D., Mandelkem, L. \& Wignall, G. D. Macromolecules, (1999), 32, 3093-3096.

Alamo, R. G., Londono, J., D. Mandelkem, L., Stehling, F. C., \& Wignall, G. D., (1994), Macromolecules, 27, 41 l-418.

Alamo, R. G., Graessley, W. W., Krishnamoorti, R., Lohse, J. D., Londono, J., D., Mandelkem, L.,

Stehling, F. C., \& Wignall, G. D., (1997), Macromolecules, 30, 561-570.

Barham, P. J., Hill, M. J., Keller, A., Rosney, C. C. A. (1998), J. Mat. Sci. Left., 1271-1278.

Graessley W. W., Krishnamoorti R., Balsara N. P., Fetters L. J., Lohse D. J., Schultz D. N., Sissano J. A. (1994), Macromolecules, 26, 1137-I 145.

Hill, M. J., Barham, P. J., Keller, A., Rosney, C. C. A. (1991), Polymer, 32 1384-1392.

Koehler W. C., (1986), Physica (Utrecht), 137B, 320-330.

Krishnamoorti, R., et al., Macromolecules, (1994), 27, 3073-2081.

Londono, J. D., Narten, A. H., Wignall, G. D., Honnell, K. G., Hsieh, E. T., Johnson, T. W., Bates, F. S. (1994) Macromolecules, 27, 2864-2873.

Mirabella, F. M. \& Ford, E.A., J. Polym. Sci., Polym., Phys., Ed., (1987), 25 777-783.

Nesarikar, A., \& Crist, B., (1994), J. Polym. Sci., B32, 641-650.

Renninger, A. L., Wicks, G.G. \& Uhlmann, D. R., (1975), J. Polym. Sci., Polym. Phys. Ed., 13, 1247-1254.

C. Schipp, M. J. Hill, P. J. Barham, V. M. Cloke, J. S, Higgins \& L. Oiazabal (1996), Polymer, 37 2291-2297.

Wignall G. D., Bates F. S. (1986), J. Appl. Cryst., 20, 28-36.

G. D. Wignall, J. D. Londono, R. G. Alamo, L. Mandelkem \& F. C. Stehling (1986), Macromolecules, 29, 5332-5339.

Wignall, G. D., Londono, J. D., Lin, J. S., Alamo, R. G., Gallante M. J. \& Mandelkem, L., (1995), Macromolecules, 28, 3156-3164. 


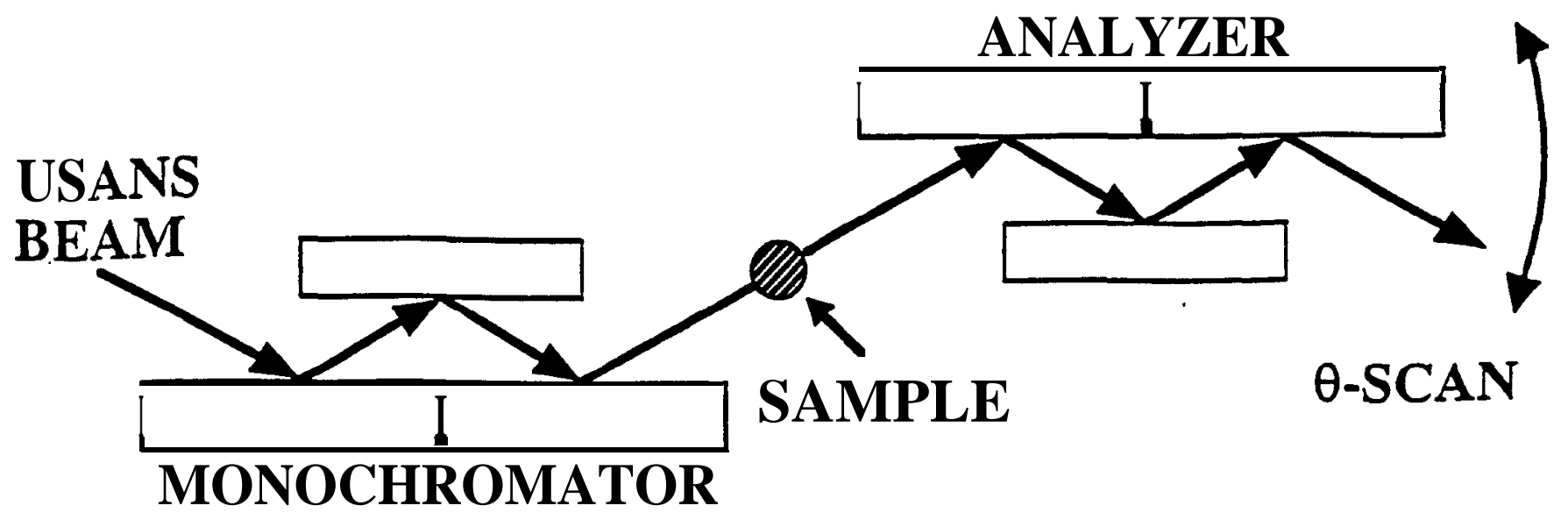

Fig. 1. Schematic layout of USANS experiment: the analyzer is rotated to perform $\theta$-scan; scattering for the sample "spreads" the beam and is recorded'as a broadened rocking curve. 


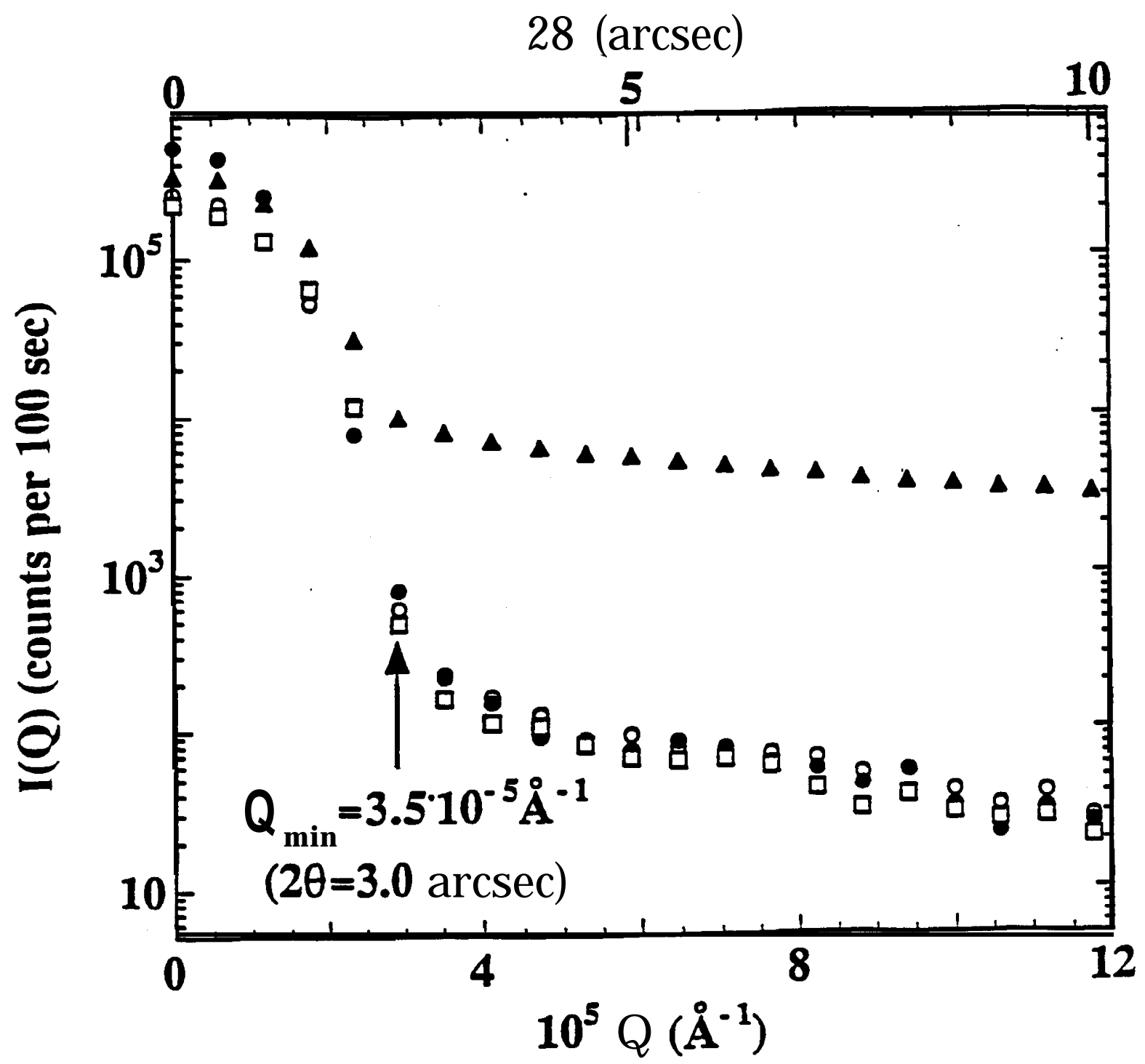

Fig. 2. Raw rocking curves from HDPE/HPB (close triangles), HDPE/LDPE (open circles),

LDPE-blank (open squares) polymer blends and from furnace with empty cell (close circles) measured in the region $0 \leq 2 \theta \leq 10$ arcsec. 


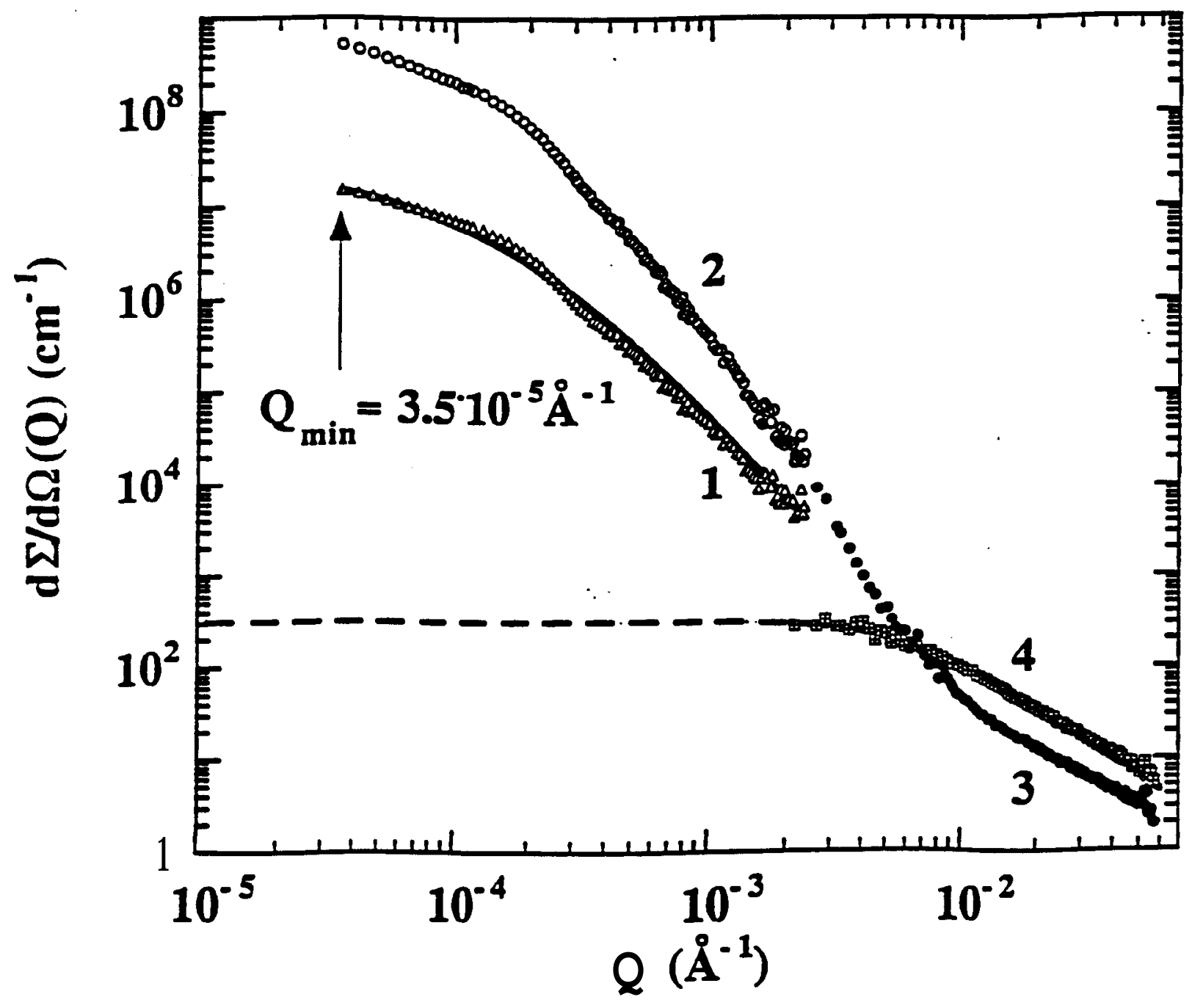

Fig. 3. Combined SANS and USANS data from HDPE/HPB and HDPE/LDPE polymer blends. USANS experimental curves 1 from HDPE/HPB sample is fitted to the Sabine-Bertram model (solid line) calculated for slit geometry. Curve 2 shows the same data transferred to point geometry by the Lake technique and overlapped with SANS data (curve 3) obtained at he $30 \mathrm{~m}$ pin-hole SANS instrument for the same HDPE/HPB blend. Curve 4 is SANS data collected from HDPE/LDPE sample fitted to the de Gennes' RPA function (dashed line). 


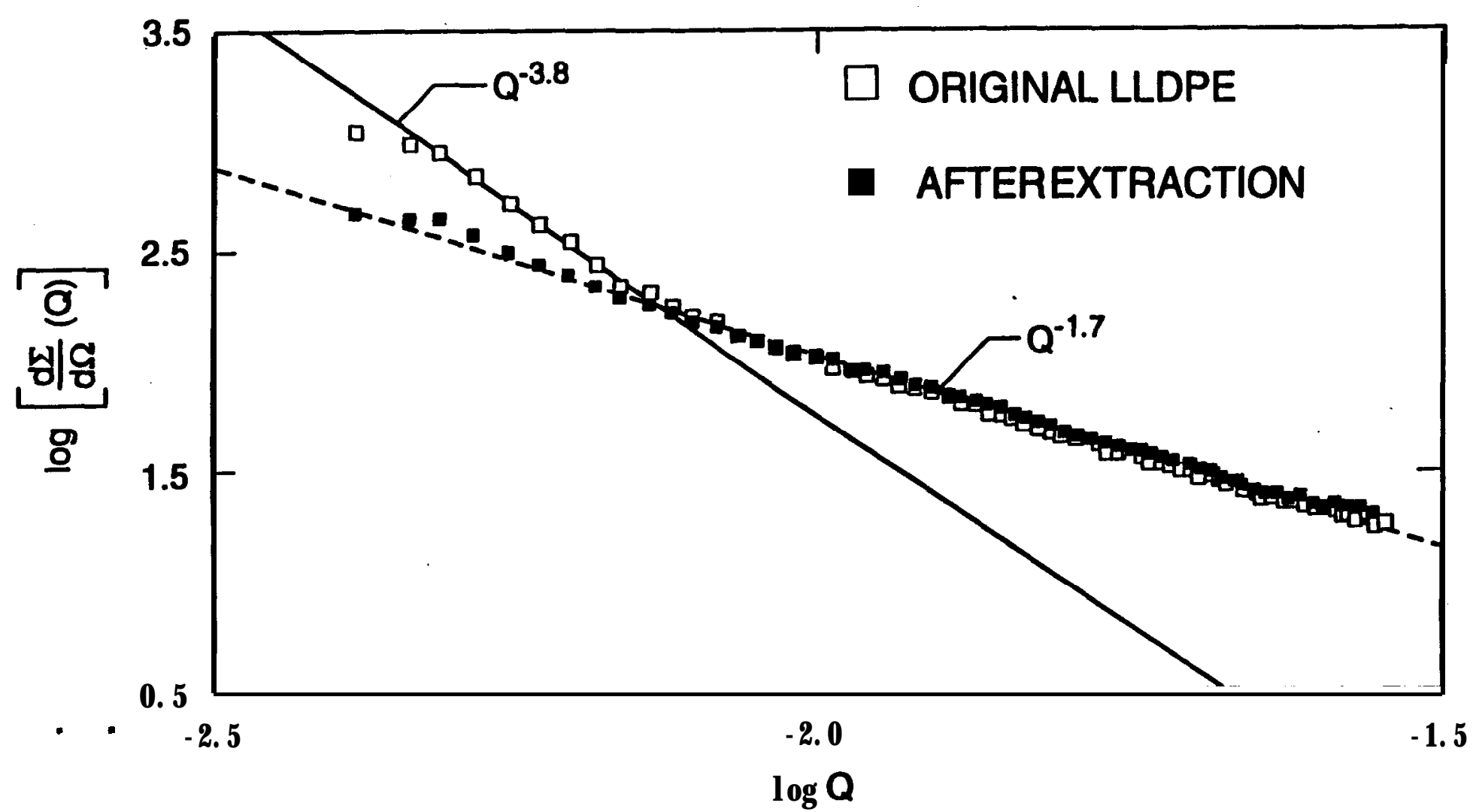

Fig /4) LOG-LOG PLOT. FOR COMPOSITIONALLY HETEROGENEOUS ETHYLENE-HEXENE COPOLYMER BEFORE AND AFTER XYLENE EXTRACTION (BOTH SAMPLES BLENDED WITH 20 WT\% HDPE-D TO PROVIDE SANS CONTRAST) 


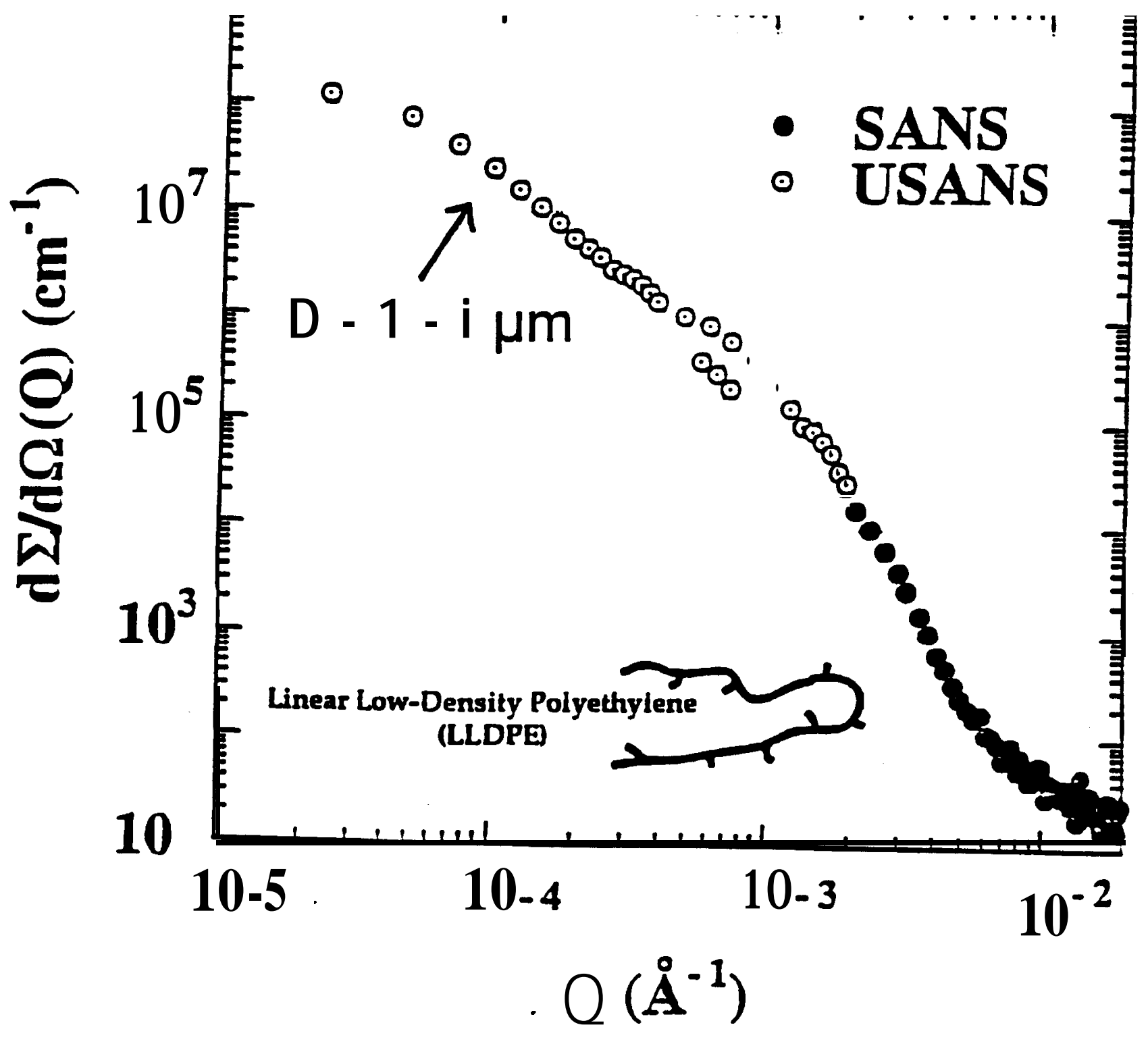

Fig (5) OVERLAP OF SANS AND USANS DATA FROM HETEROGENEOUS (ZIEGLER NATTA) LLDPE BLENDED WITH 20\% HDPE-D TO PROVIDE SANS CONTRAST 